\title{
NMDA Receptor-Dependent Regulation of Dendritic Spine Morphology by SAP102 Splice Variants
}

\author{
Bo-Shiun Chen, ${ }^{*}$ Eleanor V. Thomas, ${ }^{*}$ Antonio Sanz-Clemente, and Katherine W. Roche \\ Receptor Biology Section, National Institute of Neurological Disorders and Stroke, National Institutes of Health, Bethesda, Maryland 20892
}

\begin{abstract}
Membrane-associated guanylate kinases (MAGUKs) are major components of the postsynaptic density and play important roles in synaptic organization and plasticity. Most excitatory synapses are located on dendritic spines, which are dynamic structures that undergo morphological changes during synapse formation and plasticity. Synapse-associated protein 102 (SAP102) is a MAGUK that is highly expressed early in development and mediates receptor trafficking during synaptogenesis. Mutations in human SAP102 cause mental retardation, which is often accompanied with abnormalities in dendritic spines. However, little is known about the role of SAP102 in regulating synapse formation or spine morphology. We now find that SAP102 contains a novel NMDA receptor binding site in the $\mathrm{N}$-terminal domain, which is specific for the NR2B subunit. The interaction between SAP102 and NR2B is PDZ (postsynaptic density-95/ Discs large/zona occludens-1) domain independent and is regulated by alternative splicing of SAP102. We show that SAP102 that possesses an N-terminal insert is developmentally regulated at both mRNA and protein levels. In addition, expression of SAP102 increases synapse formation. Furthermore, the alternative splicing of SAP102 regulates dendritic spine morphology. SAP102 containing the N-terminal insert promotes lengthening of dendritic spines and preferentially promotes the formation of synapses at long spines, whereas a short hairpin RNA knockdown of the same SAP102 splice variant causes spine shrinkage. Finally, blocking NMDA receptor activity prevents the spine lengthening induced by the N-terminal splice variant of SAP102. Thus, our data provide the first evidence that SAP102 links NMDA receptor activation to alterations in spine morphology.
\end{abstract}

\section{Introduction}

Most excitatory synapses in the brain are formed on dendritic spines. The density and morphology of spines change throughout development. Several different spine shapes have been identified including thin, stubby, and mushroom spines (Bourne and Harris, 2008). Early in development, dendrites have longer and thinner filopodia that play an important role in synaptogenesis by initiating axonal contact, which subsequently leads to synapse formation (Yoshihara et al., 2009). Thin spines are short-lived, appearing and disappearing over a few days, whereas mushroom spines are relatively stable and can last for months (Holtmaat and Svoboda, 2009).

NMDA receptor (NMDAR) activity is critical in regulating dendritic spine morphology. NMDARs, localized at the postsynaptic membrane of most excitatory synapses, are tetramers composed of NR1 and NR2 subunits (Chen and Roche, 2007). Activation of NMDARs leads to an influx of calcium ions into the dendritic spine, which activates a complex signaling cascade to induce forms of synaptic plasticity, such as long-term potentia-

Received Feb. 26, 2010; revised 0ct. 11, 2010; accepted 0ct. 18, 2010.

This work was supported by the National Institute of Neurological Disorders and Stroke (NINDS) Intramural Research Program (E.V.T., B.-S.C., K.W.R.) and a NINDS Career Transition Award (E.V.T., B.-S.C.). We thank the NINDS sequencing facility and light imaging facility.

*B.-S.C. and E.V.T. contributed equally to this work.

Correspondence should be addressed to Bo-Shiun Chen, Program of Developmental Neurobiology, Institute of Molecular Medicine and Genetics, Medical College of Georgia, CA3008, 1120 15th Street, Augusta, GA 30912. E-mail: bochen@mcg.edu.

DOI:10.1523/JNEUROSCI.1034-10.2011

Copyright $\odot 2011$ the authors $\quad 0270-6474 / 11 / 310089-08 \$ 15.00 / 0$ tion (LTP) and long-term depression (LTD). Studies have shown that growth and/or enlargement of spines are associated with induction of LTP, whereas LTD-inducing stimulation causes shrinkage and/or retraction of spines (Matsuzaki et al., 2004; Nägerl et al., 2004; Okamoto et al., 2004; Zhou et al., 2004). There is evidence that NMDARs regulate spine morphology by modulating the activity of regulators of the actin cytoskeleton (Tada and Sheng, 2006). However, how NMDAR activation is linked to actin dynamics and spine morphology is not well understood.

NMDARs bind to the PSD-95 family of proteins, or membrane-associated guanylate kinases (MAGUKs), which include PSD-95, PSD-93, SAP102, and SAP97. These postsynaptic scaffolding proteins link NMDARs to the actin cytoskeleton (Elias and Nicoll, 2007). MAGUKs regulate synaptic targeting of glutamate receptors, as well as signaling and plasticity at excitatory synapses. They possess three postsynaptic density-95/Discs large/zona occludens-1 (PDZ) domains, an SH3 domain, and a catalytically inactive guanylate kinase (GK) domain. In addition, recent studies have shown that SAP97, PSD-95, and PSD-93 all have unique $\mathrm{N}$ termini expressing either a pair of cysteines that are palmitoylated or an L27 domain, which govern their role in regulating synaptic function (Schlüter et al., 2006). SAP102 does not have an L27 domain, nor is it palmitoylated. Instead, it contains an alternatively spliced region in the $\mathrm{N}$ terminus of unknown function (Müller et al., 1996).

Mutations in the human DLG3 (SAP102) gene have been reported to cause mental retardation, which is often associated with dendritic spine abnormalities (Tarpey et al., 2004; Zanni et al., 2010). However, it is not known whether SAP102 directly regu- 


\section{A

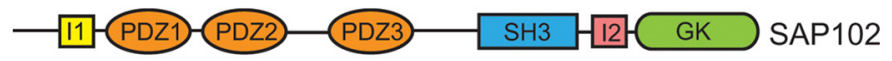

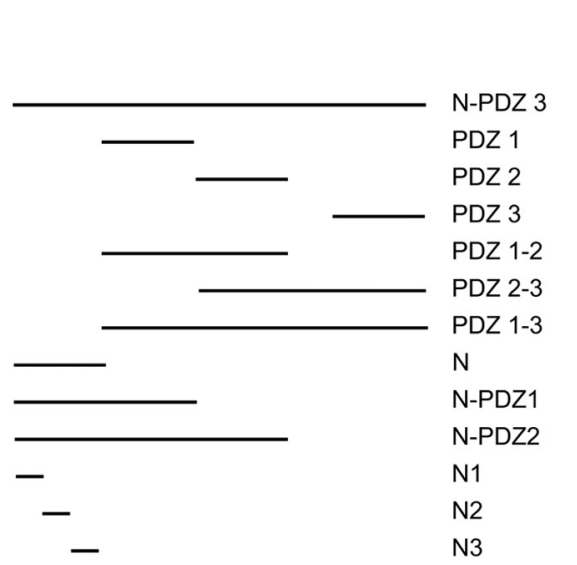

NR2B $\triangle P D Z$

$+$

$-$

$-$

$-$

$-$

-

$-$

$+$

$+$

$+$

$+$

$-$

$-$

$+$
B

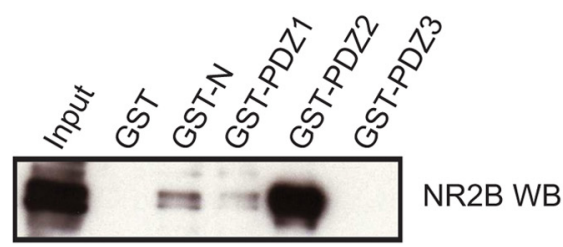

C

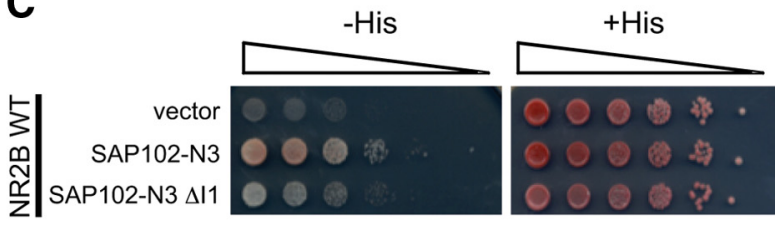

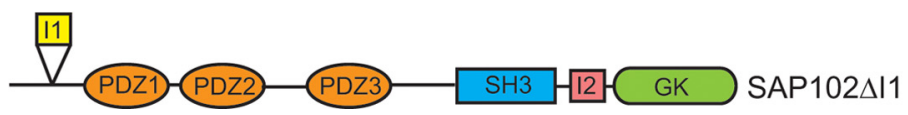

Figure 1. SAP102 contains a secondary NR2B binding site in the N terminus and the interaction is regulated by N-terminal splicing. $A$, Schematics of two naturally occurring splice variants of SAP102. SAP102 contains both I 1 and I 2 regions. SAP102 $\mathrm{I} 1$ contains an 18 aa deletion of the 11 region (depicted as a yellow box) in the N terminus (Müller et al., 1996). Various deletion constructs are shown aligned under full-length SAP102. The interaction of SAP102 constructs with the cytoplasmic tail of NR2B without PDZ ligand are shown, as measured by the yeast two-hybrid binding assay. B, Equal amounts of GST alone or GST fused to N-terminal domain, PDZ1, PDZ2, or PDZ3 were incubated with lysate of NR2B-transfected HEK-293 cells (input). After centrifugation of immobilized GST, samples were washed with buffer containing 1\% TX-100, resolved by SDS-PAGE, and immunoblotted with NR2B antibody. Equal amounts of GST protein were used in these assays, as determined by Coomassie-stained SDS-PAGE-resolved protein samples (data not shown). C, Yeast were cotransformed with LexA-NR2B and Gal4 vector, Gal4-SAP102-N3 or Gal4-SAP102-N3 $\Delta$ I1, and growth was evaluated on appropriate yeast selection medium. Results shown are 10-fold serial dilutions of yeast cells.

lates spine morphology. In the current study, we find that two naturally occurring N-terminal splice variants of SAP102 differentially bind to NR2B. Interestingly, they are associated with different types of dendritic spines. One variant specifically induces an NMDAR-dependent lengthening of dendritic spines. Thus, our findings provide evidence that SAP102 couples NMDAR activation to changes in spine morphology in an alternative splicing-dependent manner.

\section{Materials and Methods}

DNA constructs. The rat SAP102 N-PDZ 3 (amino acids 1-481), PDZ 1 (amino acids 148-232), PDZ 2 (amino acids 244-327), PDZ 3 (amino acids 404-481), PDZ 1-2 (amino acids 148-327), PDZ 2-3 (amino acids 244-481), PDZ 1-3 (amino acids 148-481), N (amino acids 1-148), N-PDZ 1 (amino acids 1-232), N-PDZ 2 (amino acids 1-327), N1 (amino acids 1-50), N2 (amino acids 51-100), and N3 (amino acids 101-148) were amplified by PCR using synthetic primers that include flanking XhoI and EcoRI recognition sequences and subcloned into the Gal4 activation domain-fusion vector pGAD10. The N-terminal, PDZ1, PDZ2, and PDZ3 domains of SAP102 were amplified by PCR using synthetic primers that include flanking EcoRI and XhoI recognition sequences and subcloned into the glutathione $S$-transferase (GST) fusion vector pGEX-6T-1 (GE Healthcare). The rat full-length SAP102 was amplified by PCR using synthetic primers that include flanking EcoRI and HindIII recognition sequences and subcloned into the p3xFLAGCMV-7.1 vector (Sigma-Aldrich). Gal4-N3 $\Delta \mathrm{I} 1$ (SAP102) and FLAGSAP102 $\Delta$ I1 were constructed by deleting the DNA fragment between amino acids 120 and 137 using site-directed mutagenesis (Stratagene). Short hairpin RNA (shRNA) oligonucleotides were inserted into FHUGW. The following shRNA targeting sequence were used for SAP102, CCAAGTCCATCGAAGCACT; I1 region-containing SAP102, CCCAGCCTATCGGTGAATG.

Yeast two-hybrid assay. The yeast two-hybrid assay was performed as described previously (Chen et al., 2006). Briefly, constructs in the LexAfusion vector pBHA (TRP1 plasmid) and the Gal4 activation domain- fusion vector pGAD10 (LEU2 plasmid) were cotransformed into L40 yeast. After transformation, the yeast were plated in synthetic complete medium lacking leucine and tryptophan (+His). Three independent yeast colonies were selected and assayed for expression of the reporter HIS3 gene in synthetic complete medium lacking leucine, tryptophan, and histidine (-His), demonstrating protein-protein interactions. 3-AT (3-aminotriazole) (10 mM) was included in the -His medium to reduce or eliminate the background transactivation. All plates were photographed after $3 \mathrm{~d}$ of incubation at $30^{\circ} \mathrm{C}$.

GST pull-down assay. GST fusion proteins were purified as described previously (Chen et al., 2006). A total of $3 \mu \mathrm{g}$ of GST fusion protein was incubated with $20 \mu \mathrm{l}$ bed volume of glutathione-Sepharose beads in 300 $\mu \mathrm{l}$ of PBS at $4^{\circ} \mathrm{C}$ for $1 \mathrm{~h}$. Input NR2B protein was obtained from the lysate of HEK-293 cells expressing NR2B. Cells were solubilized in PBS containing 1\% Triton X-100 (TX-100). Insoluble material was removed by centrifugation at $100,000 \times g$ for $20 \mathrm{~min}$. The supernatant was incubated with GST and its derivatives at $4^{\circ} \mathrm{C}$ for $4 \mathrm{~h}$. Beads were collected by centrifugation, washed three times with PBS containing 1\% TX-100, and boiled in $15 \mu \mathrm{l}$ of loading buffer. Proteins were resolved by SDS-PAGE and analyzed by immunoblotting.

RNA extraction and quantitative real-time PCR analysis. Total RNA was isolated from cortex of mice of different postnatal ages using Absolutely RNA Miniprep kit (Stratagene). cDNA synthesis was performed using random primers with AccuScript HF RT-PCR System (Stratagene) following manufacturer's instructions. The real-time PCR was performed in 7900HT Real-Time PCR System (Applied Biosystems) using Maxima SYBR Green/ROX qPCR Master Mix (Fermentas) with the primers detailed below and $40 \mathrm{ng}$ of cDNA as follows: 40 cycles at $95^{\circ} \mathrm{C}$ for $15 \mathrm{~s}, 64^{\circ} \mathrm{C}$ for $30 \mathrm{~s}$, and $72^{\circ} \mathrm{C}$ for $1 \mathrm{~min}$. Each sample was run in triplicate. Quantification was performed using the $\Delta \mathrm{CT}$ method (CtSAPCtI1). Data are presented as mean \pm SEM normalized with postnatal day $2(\mathrm{P} 2)(n=4)$. The specificity of the primers was analyzed by performing a 35 cycle PCR with FLAG-SAP102 II1, FLAG-SAP102, or cDNA from $\mathrm{P} 5$ cortex. The expected product size for common and I1-specific primers is 819 and 372 bp, respectively: I1 specific, forward, $5^{\prime}$-gctgtaagtgccccgagt- 
A

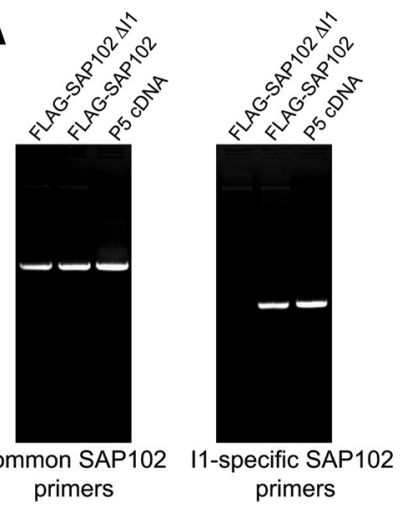

B

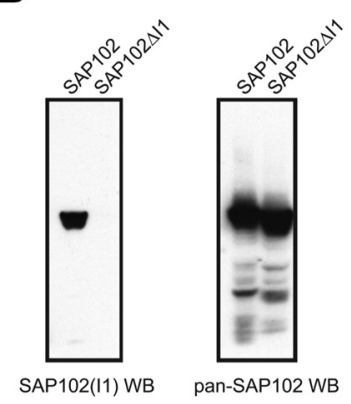

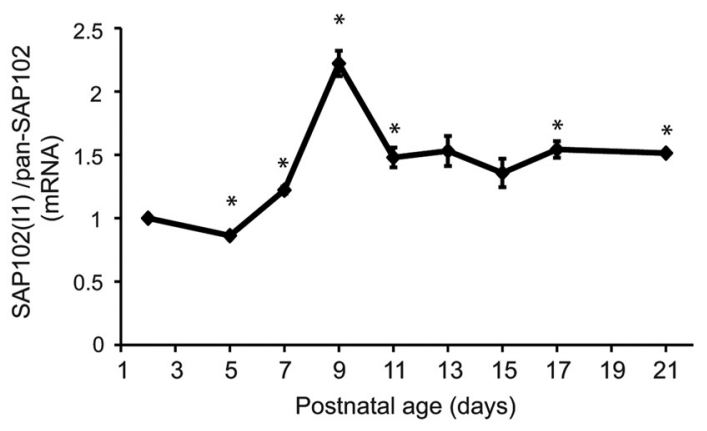

C

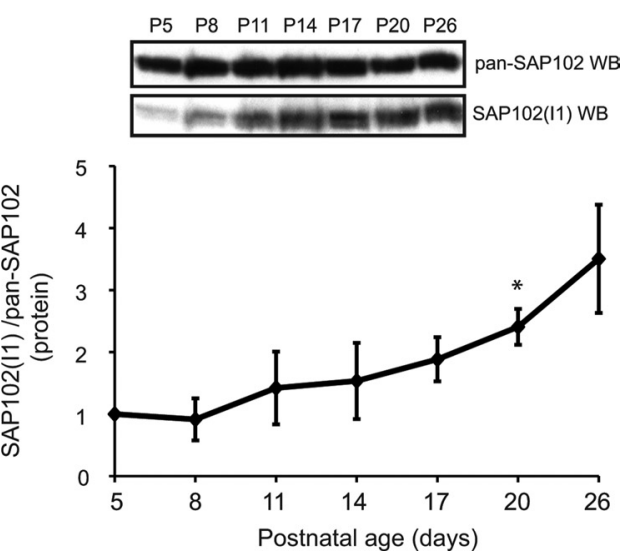

Figure 2. SAP102 splice variants are developmentally regulated. $A$, Specificity of common- and I1-specific SAP102 primers was confirmed by performing a PCR with FLAG-SAP102 11 , FLAG-SAP102, or CDNA from P5 cortex. The mRNA levels of SAP102 and SAP102(11) were measured performing real-time PCR with $40 \mathrm{ng}$ of CDNA from mouse cortex of different ages. Samples were run in triplicate $(n=4)$. Data are presented as SAP102(11)/SAP102 mean \pm SEM, normalized with P2 ( ${ }^{*} p<0.05$, one-sample $t$ test). B, HEK-293 cells were transfected with SAP102 or SAP102 11 , and immunoblots of cell lysate were probed with either panSAP102 antibody (detects all SAP102 splice variants) or with I1-specific SAP102 antibody (detects SAP102 and SAP102 DI2). C, Whole rat brain lysate was collected at various ages. The P2 fraction was isolated and resolved by $10 \%$ SDS-PAGE. Samples were immunoblotted with either pan-SAP102 antibody or with 11-specific SAP102 antibody. The experiment was repeated three times and quantified using ImageJ. A representative blot is shown in $C$. All ages were normalized to the intensity at $\mathrm{P} 5$, and a ratio of SAP102(I1) to total SAP102 was determined. Data represent means \pm SEM ( ${ }^{*} p<0.05$, one-sample $t$ test).

gctatgaggtgacc- $3^{\prime}$; I1 specific, reverse, $5^{\prime}$-cctcggggttcactaggaggggtgcag-3'; common SAP102, forward, 5'-gtatgctcccccogactatgccagc-3'; common SAP102, reverse, $5^{\prime}$-aagtcccgattagactcaatcatccctgtc- $3^{\prime}$.

Antibodies. Rabbit antibodies recognizing SAP102(I1) were generated by Pocono Rabbit Farm and Laboratory. Rabbits were immunized with a synthetic peptide Ac-CEQASPAPLLVNPEALEPS-OH corresponding to amino acids $118-135$ of SAP102. Sera were collected and affinitypurified using the antigen peptide. Monoclonal anti-FLAG, anti-NR2B, anti-tubulin, and anti-synaptophysin antibodies were purchased from Sigma-Aldrich. Pan-SAP102 antibody was purchased from NeuroMab (Antibodies, Inc.)

Immunoblots. Whole rat brain lysate was collected at various ages. The crude synaptosome (P2) fraction was prepared as described previously (Chen and Roche, 2009) except $5 \mathrm{~mm}$ iodoacetamine was added in the PBS buffer in addition to protease inhibitor mixture (Roche Applied Science) to prevent SAP102(I1) protein degradation. The P2 lysate was resolved by $10 \%$ SDS-PAGE and analyzed by Western blot with either pan-SAP102 antibody (detects all SAP102 splice variants) or with I1specific SAP102 antibody. The experiment was repeated three times and quantified using ImageJ. It is important to note that SAP102(I1) was extremely unstable, resulting in variation in our data. All ages were normalized to the intensity at P5 and then a ratio of SAP102(I1) to total SAP102 was determined. The average ratio is shown.

Neuronal cultures and transfections. Primary hippocampal cultures were prepared from embryonic day 17 (E17) to E19 Sprague Dawley rats.
Dissociated neurons were plated on poly-Dlysine-coated coverslips in Neurobasal medium supplemented with B27 and L-glutamine. Hippocampal neurons were transfected at $12 \mathrm{~d}$ in vitro (DIV) using Lipofectamine (Lipofectamine 2000; Invitrogen). Neurons were fixed at DIV14 in 4\% PFA for 15 min, permeabilized with $0.25 \%$ TX-100/PBS, and blocked in $10 \%$ normal donkey serum/PBS. Cells were incubated with primary antibodies against FLAG in PBS/3\% normal donkey serum, washed, and incubated with secondary antibodies (Alexa 488 or 568 ) in PBS/3\% normal donkey serum against mouse IgG1. The cells were mounted onto coverslips (Prolong Antifade kit; Invitrogen).

Immunofluorescence experiments. Primary hippocampal neurons were treated with APV $(100 \mu \mathrm{M})$ or ifenprodil $(3 \mu \mathrm{M})$ at DIV13 and fixed and stained $24 \mathrm{~h}$ later. Neurons were fixed and stained with anti-FLAG antibody as previously described. Neurons transfected with green fluorescent protein (GFP) and FLAGSAP102(I1) or FLAG-SAP102DI1 were stained with anti-FLAG (data not shown) and antisynaptophysin antibodies. Colocalization of dendritic spines expressing GFP (and transfected with FLAG-SAP102) with synaptophysin was determined manually. Synaptic spines were defined as dendritic spines overlapping with synaptophysin. Long spines were defined as $>2 \mu \mathrm{m}$.

Image acquisition and analysis. Images were acquired using a Zeiss LSM 510 confocal microscope. Neurons were selected randomly from each coverslip with the viewer blinded to the experimental condition. At least three independent experiments were performed per condition, and the number of neurons for each condition is indicated in the corresponding figure legend. Three $20 \mu \mathrm{m}$ sections of dendrite were selected from each neuron for analysis. Images were collected using a $63 \times$ oilimmersion objective, and $Z$-stacked images were analyzed using Zeiss LSM 510 software. Dendritic spines were measured with the viewer blinded to the experimental condition. Length was measured by manually drawing a line from the base of the spine neck to the furthest point at the end of the spine. Measurements were analyzed in Microsoft Excel, and statistical significance was determined by Student's $t$ test. The cumulative frequency plots were constructed by sorting the length or width in bins of 0.5 or $0.3 \mu \mathrm{m}$ size and using a discrete variable on the horizontal axis. The ordinal axis was labeled with percentages. $N=$ number of neurons.

\section{Results}

\section{SAP102, but not PSD-95, interacts with NR2B lacking the} PDZ ligand

The interaction between NMDARs and the PSD-95 family of proteins is critical for the localization and clustering of receptors at the postsynaptic membrane (Wenthold et al., 2003). It has been accepted that the PDZ domains of PSD-95 family members are the essential binding sites for NR2 subunits of NMDARs. However, NR2A preferentially binds to PSD-95 and NR2B preferentially binds to SAP102 (Sans et al., 2000). Because NR2A and $\mathrm{NR} 2 \mathrm{~B}$ share an identical PDZ binding motif in the extreme C terminus, the molecular basis for this differential binding is not clear. Therefore, we investigated whether PSD-95 could bind to the NR2 subunits lacking the PDZ ligand, which might reveal an 
additional binding site. We used a yeast two-hybrid binding assay to examine the protein-protein interactions. Consistent with previous results, NR2B bound exclusively to the N-terminal half of PSD-95 (PSD-95-N) and SAP102 (SAP102-N), which contain the PDZ domains, and truncation of the PDZ ligand of NR2B (NR2B $\triangle \mathrm{PDZ}$ ) disrupted binding to PSD-95 (supplemental Fig. S1, available at www.jneurosci.org as supplemental material). However, SAP102 still bound to NR2B even after truncation of the PDZ binding motif and the interaction required the N-terminal half of SAP102 (supplemental Fig. S1, available at www. jneurosci.org as supplemental material). Together, these data indicate that SAP102 contains an additional NR2B binding site in the $\mathrm{N}$ terminal half of SAP102 that is not conserved in PSD-95.

\section{SAP102 splicing regulates the}

\section{PDZ-independent binding to NR2B}

To identify the secondary binding site, we delimited the SAP102-N sequences responsible for the NR2B $\triangle \mathrm{PDZ}$ interaction. Through a series of truncation studies, we found that the N3 domain of SAP102 (amino acids 101-148) is the minimum region required for the non-PDZ interaction (Fig. 1A) (data not shown). We also evaluated the interaction using an in vitro binding assay using GST fusion proteins containing the N-terminal, PDZ1, PDZ2, or PDZ3 domain of SAP102. The input protein for these assays was generated from the lysate of NR2B-transfected HEK-293 cells. In agreement with previous studies, NR2B displayed robust binding to PDZ2, weak binding to PDZ1, and negligible binding to PDZ3 (Fig. 1B) (Niethammer et al., 1996). Consistent with our yeast two-hybrid data, NR2B interacted with the N-terminal domain of SAP102, although the binding was not as robust as with PDZ2.

Three splice variants of SAP102 have been identified (Müller et al., 1996). In addition to the first identified SAP102, one splice variant contains an 18 aa deletion in the $\mathrm{N}$ terminus (amino acids 120-137), which is called the I1 region (Fig. 1A), and another contains a 14 aa deletion between the SH3 and GK domains (amino acids 626-639) called the I2 region. Interestingly, the I1 region coincides with the SAP102 N3 domain that contains the secondary NR2B binding site. To explore whether I1 splicing of SAP102 has any effect on NR2B binding, we made a SAP102 N3 construct lacking the I1 region to mimic I1 splicing and analyzed protein binding using the yeast two-hybrid binding assay. We found that deletion of the I1 region of SAP102 N3 disrupted the PDZ-independent binding to NR2B (Fig. 1C).

\section{Developmental regulation of SAP102 splice variants}

Studies have shown that the unique $\mathrm{N}$-terminal regions of PSD-95 and SAP97 have different roles in regulating synaptic AMPA receptor function (Schlüter et al., 2006). Therefore, we characterized the developmental expression of the SAP102 splice variants containing the I1 region (SAP102 and SAP102 $\Delta \mathrm{I} 2$ ). In this study, we use SAP102 to indicate the isoform containing both
I1 and I2. We first analyzed the ratio of the I1 region-containing SAP102 [SAP102(I1)] to total SAP102 expression at the mRNA level using real-time PCR. We observed a dramatic increase in SAP102(I1) compared with total SAP102 starting at P7 and reaching a peak at $\mathrm{P9}$. Subsequently, the relative expression of the I1-containing splice variants decreases somewhat and then plateaus (Fig. 2A). The fact that peak expression of SAP102(I1) at the mRNA level occurs between P7 and P11, a time critical for the development of dendritic filopodia (Lendvai et al., 2000), suggests that SAP102(I1) plays an important role in neuronal development. We also raised an antibody specifically recognizing the I1 region-containing SAP102 [SAP102(I1)] (Fig. 2B). Western blot analysis was performed using homogenized rat brain lysate measuring total levels of SAP102 and SAP102(I1), which show that SAP102(I1) increases during the first month of life, as total SAP102 levels increase (Fig. 2C). Both total SAP102 and SAP102(I1) levels decrease during adulthood (data not shown). Quantification of the Western blots at P20 showed that the ratio of SAP102(I1) to total SAP102 levels increases significantly during the first month of life, supportive of the mRNA findings.

Expression of SAP102 enhances synapse formation on spines Overexpression of PSD-95 drives maturation of excitatory synapses (El-Husseini et al., 2000a). However, whether SAP102 has any effect on synapse number or dendritic spine morphology during synaptogenesis is not known. We therefore examined colocalization of SAP102 with synaptophysin, a presynaptic marker 
A
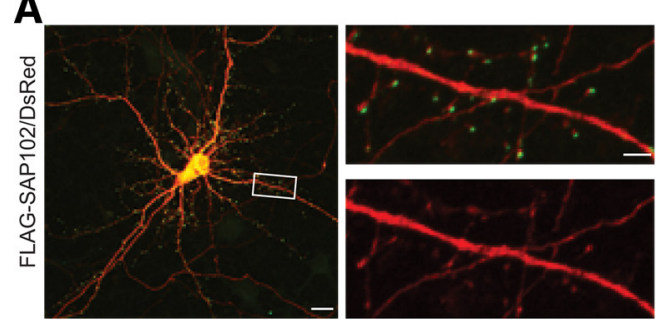

C

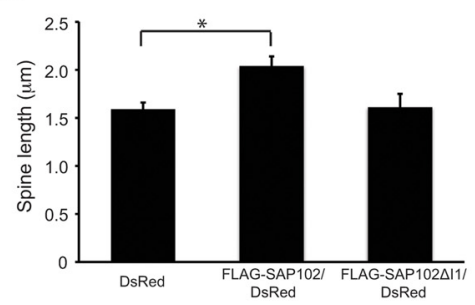

B
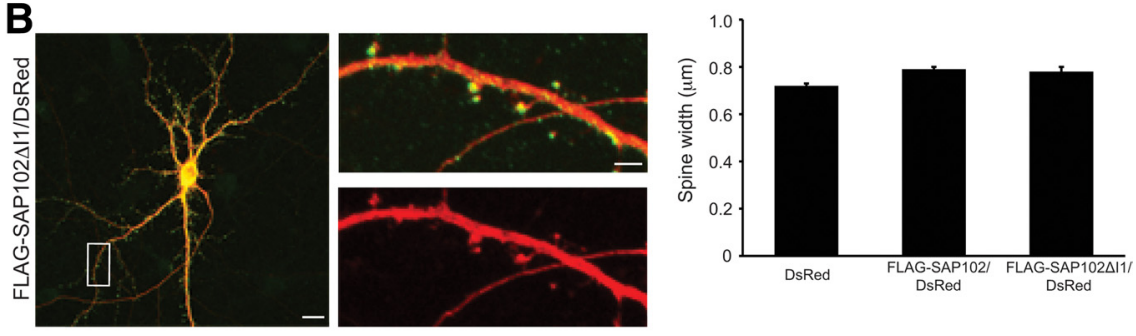

D
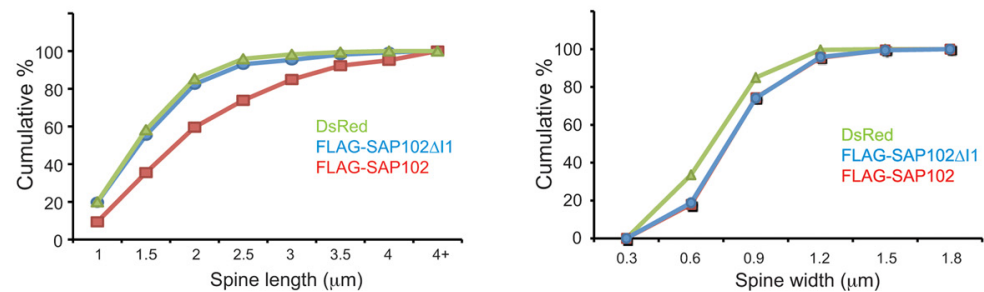

Figure 4. SAP102 regulates spine morphology. $\boldsymbol{A}, \boldsymbol{B}$, Primary hippocampal neurons (DIV12) were transfected with FLAGSAP102/DsRed or FLAG-SAP102 I1/DsRed. At DIV 14, they were fixed and labeled with anti-FLAG antibody (green). Dendrites on neurons transfected with FLAG-SAP102 had longer protrusions than those transfected with FLAG-SAP102AI1/DsRed or DsRed only. $C, D$, Dendritic spines were quantified by measuring DsRed signal using LSM510 Meta software. Data represent means \pm SEM ( $n=10$ neurons per condition from 3 independent cultures; $20-30$ spines $/$ neuron; ${ }^{*} p=0.004, t$ test with Bonferroni's correction after ANOVA). Cumulative frequency plots of spine length and spine width are shown. Scale bars: left, $10 \mu \mathrm{m}$; right, $2 \mu \mathrm{m}$.

in primary hippocampal neurons (DIV14) expressing FLAGtagged SAP102 or SAP102 II1. We coexpressed GFP or DsRed to visualize the morphology of transfected neurons and stained with anti-FLAG to assess SAP102 expression levels. Synaptic spines were identified by colocalization of the spine heads with synaptophysin puncta. Interestingly, both splice variants of SAP102 promoted synapse formation (Fig. $3 A-C$ ). Dendritic spines on these neurons were more likely to form synapses than those on neurons expressing only GFP $(61.1 \pm 3.8 \%$ of SAP 102 and $62.9 \pm$ $3.6 \%$ of SAP $102 \Delta(\mathrm{I} 1)$-transfected spines formed synapses, whereas only $44.1 \pm 5.4 \%$ of spines transfected with GFP formed synapses) (Fig. 3D). To ensure that the FLAG tag did not affect our data, we also used SAP102 or SAP102 $\Delta$ I1 without the FLAG-tag in these experiments and found similar effects (data not shown).

\section{Expression of SAP102 splice variants differentially affects} spine morphology

We next examined spine morphology in primary hippocampal neurons (DIV14) expressing FLAG-tagged SAP102 or SAP102 $\mathrm{I} 1$. Neurons expressing FLAG-SAP102 had significantly longer dendritic spines, whereas the spine length of FLAG-SAP102 $\mathrm{I} 1$ expressing neurons was indistinguishable from DsRed alone (Fig. 4A,B). The mean length of dendritic spines was increased in FLAG-SAP102-transfected DIV14 neurons (from $1.59 \pm 0.07$ to $2.04 \pm 0.10 \mu \mathrm{m}$ ) (Fig. $4 C$; supplemental Fig. S2, available at www.jneurosci.org as supplemental material). Spine width did not differ between SAP102/DsRed-, SAP102 $\Delta$ I1/DsRed-, and DsRed-expressing neurons (Fig. 4C). Cumulative frequency plots of spine length revealed that SAP102 overexpression resulted in a rightward shift in spine length, whereas overexpression of SAP102 $\mathrm{I} 1$ exhibited a similar pattern to plots of DsRed alone (Fig. $4 D$ ), indicating that SAP102 expression, but not SAP102 $\Delta \mathrm{I} 1$, substantially increased the proportion of long spines in our experiments. No significant difference in the cumulative frequency of spine width was observed (Fig. 4D). In addition, the spine density was not changed significantly in neurons expressing SAP102 or SAP102 $\mathrm{I} 1$ (data not shown).

To investigate whether enhanced synapse formation by both splice variants of SAP102 correlates with their effects on spine length, we analyzed the lengths of synaptic spines induced by expression of SAP 102 or SAP $102 \Delta \mathrm{I} 1$. We found SAP 102 preferentially promoted the formation of synapses at long spines [48.4 $\pm 5.9 \%$ compared with those for SAP $102 \Delta \mathrm{I} 1$ $(16.6 \pm 3.7 \%)$ and GFP $(21.5 \pm 4.7 \%)]$ (Fig. 3E). Long spines were defined as spines $>2 \mu \mathrm{m}$. Although SAP102 $\Delta \mathrm{I} 1$ promoted synapse formation, it did not selectively increase the length of any particular subset of spines. Instead, it uniformly increased synapse formation at spines of all lengths. The distribution of synaptic spines in SAP102 $\Delta \mathrm{I} 1 / \mathrm{GFP}$-expressing neurons and GFP-expressing neurons was similar, whereas SAP102 exhibited a distinct preference for long synaptic spines compared with both conditions (Fig. $3 F$ ).

To investigate whether endogenous SAP102 plays a role in regulating spine morphology, we generated shRNA to specifically knock down expression of I1 region-containing SAP102. We tested the specificity of the shRNA by coexpression with SAP102 or SAP102 $\Delta$ I1 in HEK-293 cells. SAP102 I1 shRNA selectively knocked down expression of SAP102 and had no effect on SAP102 $\Delta$ I1 expression. In contrast, SAP102 shRNA knocked down both SAP102 and SAP102 $\Delta \mathrm{I} 1$ expression (Fig. 5A). We then measured the effect of SAP102 I1 shRNA on the expression of I1 region-containing SAP102 in primary hippocampal neurons infected with lentivirus expressing SAP102 I1 shRNA. SAP102 I1 shRNA efficiently reduced expression of I1 regioncontaining SAP102 and lentivirus expressing a control scrambled shRNA had no effect (Fig. 5B). To examine the effect of SAP102 I1 shRNA, we infected neurons with lentivirus at DIV6 and expressed DsRed to visualize the spine morphology at DIV14. Neurons expressing SAP102 I1 shRNA had significantly shorter dendritic spines compared with the spine length of control shRNA-expressing neurons (Fig. 5C,D). Cumulative frequency plots of spine length revealed that SAP102 knockdown resulted in a leftward shift in spine length (Fig. 5E), demonstrating that I1 region-containing SAP102 regulates spine morphology.

\section{SAP102-induced spine elongation is NMDAR activity dependent}

NMDAR activity has been shown to regulate spine morphology (Tada and Sheng, 2006). To determine whether the effect of SAP102 on dendritic spine morphology is NMDAR activity de- 
pendent, we pharmacologically blocked NMDARs using $100 \mu \mathrm{M}$ APV or by blocking NR2B-containing receptors using 3 $\mu \mathrm{M}$ ifenprodil. Blocking NMDARs using APV or ifenprodil completely disrupted the lengthening of dendritic spines observed in neurons expressing SAP102/ GFP (Fig. 6A, $B, E$ ). Blocking NMDARs had no effect on average spine length in neurons expressing SAP102 $\Delta \mathrm{I} 1 / \mathrm{GFP}$ or GFP alone (Fig. 6C-E). There was no difference in average spine length of neurons coexpressing DsRed versus GFP in SAP102 or SAP102 $\mathrm{I} 1$ expressing neurons. Cumulative frequency plots showed a similar lengthening of dendritic spines when SAP102 was overexpressed using both fluorophores (supplemental Fig. S2, available at www.jneurosci.org as supplemental material).

\section{Discussion}

In the present study, we identified a distinct role for SAP102 in regulating synapse number and spine morphology. First, we identify a secondary NR2B binding site in the $\mathrm{N}$ terminus of SAP102, which is regulated by $\mathrm{N}$-terminal splicing of SAP102. Second, we show that expression of SAP102(I1), which contains the newly identified NR2B binding site, is developmentally regulated. Third, we find that expression of SAP102 or SAP102 $\Delta \mathrm{I} 1$ increases synapse formation, with SAP102 preferentially promoting the formation of synapses at long spines. Finally, SAP102 expression promotes lengthening of dendritic spines, which is dependent on the activity of NMDARs.

Morphological changes of dendritic spines at excitatory synapses have emerged as a key mechanism underlying plasticity of brain function. Studies have shown that synaptic properties including number and type of receptors, calcium dynamics, and synaptic plasticity are strongly associated with changes in dendritic spine morphology, which are regulated by changes in the plasma membrane linkage with the underlying actin cytoskeleton. Dynamic remodeling of the actin cytoskeleton is mainly modulated by the Rho family of GTPases (Newey et al., 2005). Among Rho GTPases, RhoA induces the shrinkage and loss of dendritic spines. In contrast, Rac and Cdc42 promote growth and/or stability of spines. Many morphological changes of dendritic spines are dependent on NMDAR activity. However, how activation of NMDARs leads to morphological changes of dendritic spines is less clear.

PSD-95 and other scaffolding proteins at the postsynaptic density, such as Shank and Homer, have been shown to influence spine morphology. These synaptic proteins are bound to NMDARs directly or indirectly, and transduce extracellular signals to regulate the actin cytoskeleton, presumably through their interaction with other effectors. For example, PSD-95 binds to synaptic GTPase activating proteins (SynGAPs) and A-kinase anchoring protein 79/150 (AKAP79/150) and determines their synaptic localization (Kim et al., 1998; Colledge et al., 2000). Synaptic AKAP79/150, which binds to SH3 and GK domains of
B

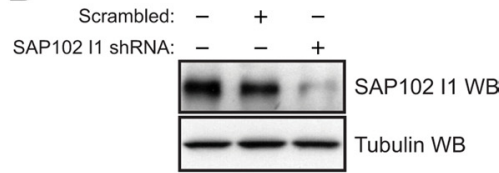

D

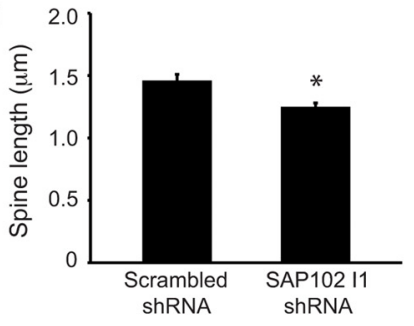

E

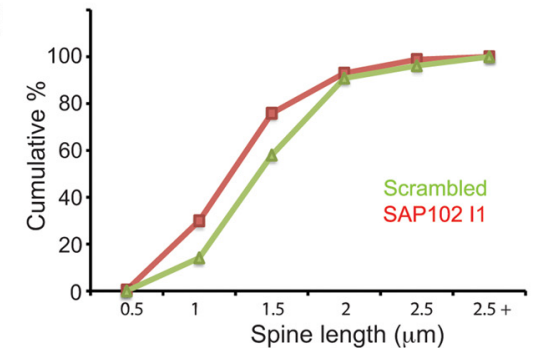

Figure 5. shRNA knockdown of endogenous I1-containing SAP102 causes spine shrinkage. $\boldsymbol{A}$, HEK-293 cells were cotransfected from three independent cultures; $20-30$ spines/neuron; ${ }^{*} p<0.01$, Student's $t$ test). Cumulative frequency plot of spine length is

PSD-95, is regulated by NMDARs through calcineurin activation and remodeling of dendritic actin (Gomez et al., 2002). In contrast, SynGAP, which interacts with the PDZ domains of PSD-95, links Rho GTPase to downstream effectors to regulate spine morphology (Carlisle et al., 2008). We now show that SAP102 containing an alternatively spliced $\mathrm{N}$-terminal insert induces lengthening of dendritic spines, which is associated with increased synapse formation at long spines. In addition, the SAP102-dependent lengthening of dendritic spines requires NMDAR activity. Because of the domain similarity between PSD-95 and SAP102, it is likely that SAP102 binds to the same effectors of PSD-95 that regulate the actin cytoskeleton. It has been shown that both PSD-95 and SAP102 bind to SynGAP via their PDZ domains, although it is not known whether SAP102 binds to AKAP79/150 (Kim et al., 1998). NMDAR activity is required for activation of a SynGAP downstream effector, Rac (Tolias et al., 2005; Xie et al., 2007). Interestingly, blocking Rac activity has been shown to transform a subpopulation of spines into long, thin filopodia-like protrusions (Tashiro and Yuste, 2004).

MAGUKs play an important role in regulating synaptic structure and plasticity. In addition to clustering receptors at the postsynaptic density, MAGUKs act as scaffolding proteins and organize various signal transduction cascades at dendritic spines. Although each MAGUK protein seems to have unique properties, they can functionally compensate for one another. For instance, double-mutant analysis of PSD-95 and SAP102 indicates that they have overlapping and specific functions (Cuthbert et al., 2007). Although extensive efforts have focused on defining the 

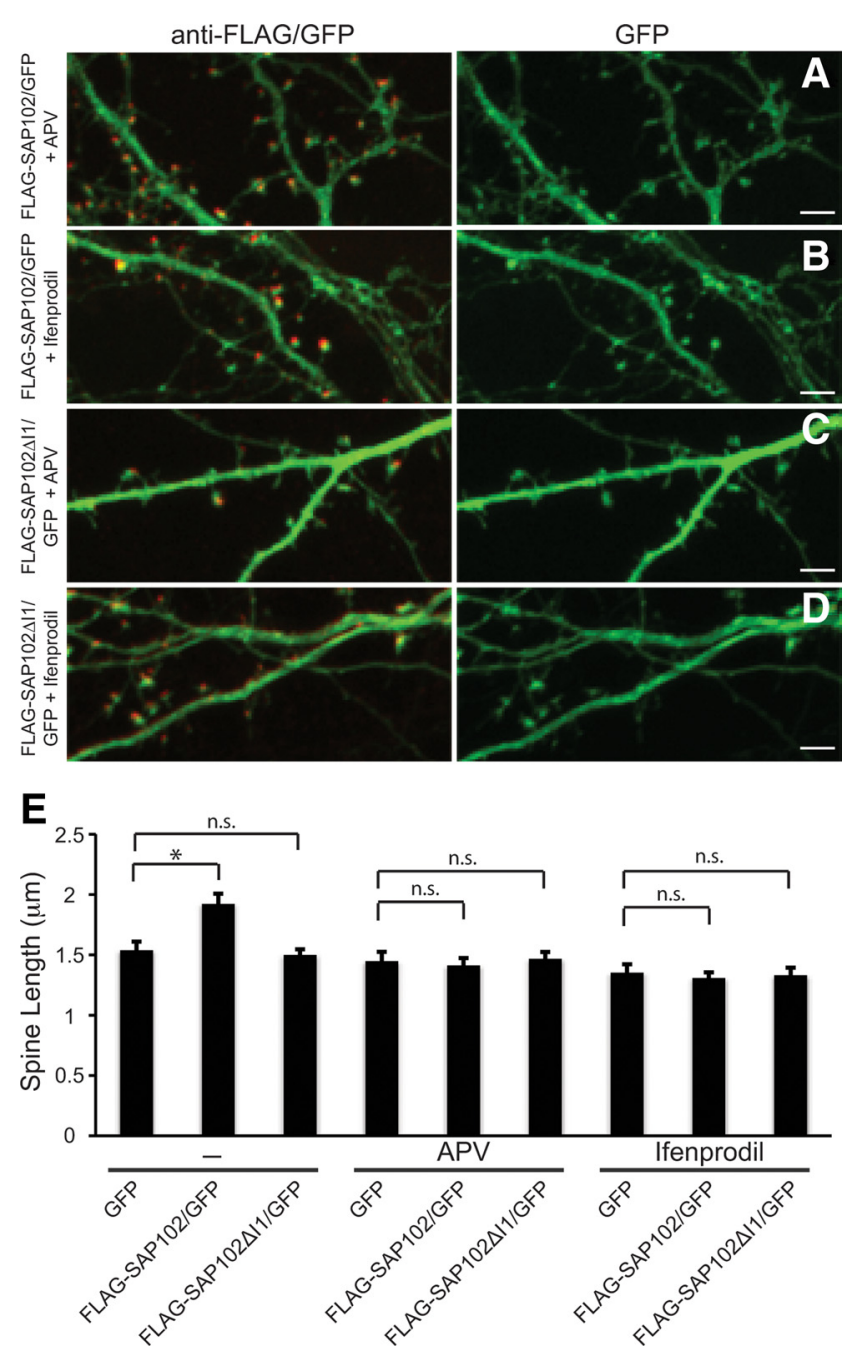

Figure 6. NMDAR activity regulates the SAP102 dependent lengthening of dendritic spines. $A-D$, Primary hippocampal neurons (DIV12) were transfected with FLAG-SAP102/GFP or FLAGSAP102 $\Delta 11 /$ GFP and were treated with either $100 \mu \mathrm{m}$ APV or $3 \mu \mathrm{m}$ ifenprodil after $24 \mathrm{~h}$. The neurons were fixed and labeled with anti-FLAG antibody at DIV14. $E$, Average dendritic spine length of transfected neurons was measured using LSM510 software. The control, APV, and ifenprodil results were treated separately. Data represent means \pm SEM $[n=10$ neurons for GFP, SAP102/GFP, and SAP102 $\Delta 11 /$ GFP; $n=9$ neurons for GFP/APV; $n=8$ neurons for GFP/ ifenprodil, SAP102/APV, SAP102/ifenprodil, SAP102 $\Delta$ I1/APV, and SAP102 $\Delta$ I1/ifenprodil; ${ }^{*} p<0.001$ (control group), $t$ test with Bonferroni's correction after ANOVA]. n.s. indicates $p>$ 0.05. Both APV and ifenprodil treatment prevented the effect observed with FLAG-SAP102. Scale bars, $2 \mu \mathrm{m}$.

synaptic function of PSD-95, there are relatively few studies on SAP102, which is highly expressed in the early postnatal period (Sans et al., 2000). Data suggest that SAP102 is the dominant MAGUK for trafficking and anchoring NMDARs at synapses during early postnatal development (Sans et al., 2003; Washbourne et al., 2004). PSD-95 expression increases later and has been shown to be involved in maturation and stabilization of excitatory synapses (El-Husseini et al., 2000a). Our findings indicate that peak expression of SAP102 containing I1 at the mRNA level occurs between P7 and P11, which coincides with a time critical for the development of dendritic filopodia (Lendvai et al., 2000), suggesting that SAP102 may play an important role in neuronal development and synaptogenesis. These results are in agreement with recent findings that SAP102 is primarily responsible for synaptic trafficking of AMPA and NMDA receptors during synaptogenesis (Elias et al., 2008).
Intriguingly, the protein ratio of SAP102(I1)/SAP102 remains elevated during the first month of life, whereas the mRNA ratio decreases at P11. This could be explained by differences in rates of protein turnover.

The N-terminal region of PSD-95 contains two cysteines that are palmitoylated and the palmitoylation targets PSD-95 to the postsynaptic membrane (Topinka and Bredt, 1998; Craven et al., 1999). In contrast, SAP102 is not palmitoylated. Instead, its $\mathrm{N}$-terminal cysteines form a zinc-binding motif (El-Husseini et al., 2000b). In addition, the N-terminal region of SAP102 contains an alternatively spliced I1 region including an additional 18 aa (Müller et al., 1996). In this study, we found that the alternative splicing of SAP102 serves as a molecular mechanism to control NMDAR interactions and spine morphology. Sequence examination of these 18 aa does not reveal homology to any identified domain or motif. How does the inclusion of I1 in SAP102 regulate the lengthening of dendritic spines? The clue might come from the secondary PDZ-independent interaction between SAP102 and NR2B in that the interaction is regulated by SAP102 I1 splicing. Evidence has accumulated that SAP102 and NR2B interact physically and functionally (van Zundert et al., 2004). Our data now provide a molecular basis for the preferential binding of NR2B to SAP102 that has been reported previously (Sans et al., 2000). In summary, we have established molecular links between NMDA receptor activity, MAGUK binding, and spine dynamics providing insight into a SAP102-dependent role in synaptic function.

\section{References}

Bourne JN, Harris KM (2008) Balancing structure and function at hippocampal dendritic spines. Annu Rev Neurosci 31:47-67.

Carlisle HJ, Manzerra P, Marcora E, Kennedy MB (2008) SynGAP regulates steady-state and activity-dependent phosphorylation of cofilin. J Neurosci 28:13673-13683.

Chen BS, Roche KW (2007) Regulation of NMDA receptors by phosphorylation. Neuropharmacology 53:362-368.

Chen BS, Roche KW (2009) Growth factor-dependent trafficking of cerebellar NMDA receptors via protein kinase B/Akt phosphorylation of NR2C. Neuron 62:471-478.

Chen BS, Braud S, Badger JD 2nd, Isaac JT, Roche KW (2006) Regulation of NR1/NR2C N-methyl-D-aspartate (NMDA) receptors by phosphorylation. J Biol Chem 281:16583-16590.

Colledge M, Dean RA, Scott GK, Langeberg LK, Huganir RL, Scott JD (2000) Targeting of PKA to glutamate receptors through a MAGUK-AKAP complex. Neuron 27:107-119.

Craven SE, El-Husseini AE, Bredt DS (1999) Synaptic targeting of the postsynaptic density protein PSD-95 mediated by lipid and protein motifs. Neuron 22:497-509.

Cuthbert PC, Stanford LE, Coba MP, Ainge JA, Fink AE, Opazo P, Delgado JY, Komiyama NH, O’Dell TJ, Grant SG (2007) Synapse-associated protein $102 /$ dlgh 3 couples the NMDA receptor to specific plasticity pathways and learning strategies. J Neurosci 27:2673-2682.

El-Husseini AE, Schnell E, Chetkovich DM, Nicoll RA, Bredt DS (2000a) PSD-95 involvement in maturation of excitatory synapses. Science 290:1364-1368.

El-Husseini AE, Topinka JR, Lehrer-Graiwer JE, Firestein BL, Craven SE, Aoki C, Bredt DS (2000b) Ion channel clustering by membraneassociated guanylate kinases. Differential regulation by N-terminal lipid and metal binding motifs. J Biol Chem 275:23904-23910.

Elias GM, Nicoll RA (2007) Synaptic trafficking of glutamate receptors by MAGUK scaffolding proteins. Trends Cell Biol 17:343-352.

Elias GM, Elias LA, Apostolides PF, Kriegstein AR, Nicoll RA (2008) Differential trafficking of AMPA and NMDA receptors by SAP102 and PSD-95 underlies synapse development. Proc Natl Acad Sci USA 105:20953-20958.

Gomez LL, Alam S, Smith KE, Horne E, Dell'Acqua ML (2002) Regulation of A-kinase anchoring protein 79/150-cAMP-dependent protein kinase 
postsynaptic targeting by NMDA receptor activation of calcineurin and remodeling of dendritic actin. J Neurosci 22:7027-7044.

Holtmaat A, Svoboda K (2009) Experience-dependent structural synaptic plasticity in the mammalian brain. Nat Rev Neurosci 10:647-658.

Kim JH, Liao D, Lau LF, Huganir RL (1998) SynGAP: a synaptic RasGAP that associates with the PSD-95/SAP90 protein family. Neuron 20:683-691.

Lendvai B, Stern EA, Chen B, Svoboda K (2000) Experience-dependent plasticity of dendritic spines in the developing rat barrel cortex in vivo. Nature 404:876-881.

Matsuzaki M, Honkura N, Ellis-Davies GC, Kasai H (2004) Structural basis of long-term potentiation in single dendritic spines. Nature 429:761-766.

Müller BM, Kistner U, Kindler S, Chung WJ, Kuhlendahl S, Fenster SD, Lau LF, Veh RW, Huganir RL, Gundelfinger ED, Garner CC (1996) SAP102, a novel postsynaptic protein that interacts with NMDA receptor complexes in vivo. Neuron 17:255-265.

Nägerl UV, Eberhorn N, Cambridge SB, Bonhoeffer T (2004) Bidirectional activity-dependent morphological plasticity in hippocampal neurons. Neuron 44:759-767.

Newey SE, Velamoor V, Govek EE, Van Aelst L (2005) Rho GTPases, dendritic structure, and mental retardation. J Neurobiol 64:58-74.

Niethammer M, Kim E, Sheng M (1996) Interaction between the C terminus of NMDA receptor subunits and multiple members of the PSD-95 family of membrane-associated guanylate kinases. J Neurosci 16:21572163.

Okamoto K, Nagai T, Miyawaki A, Hayashi Y (2004) Rapid and persistent modulation of actin dynamics regulates postsynaptic reorganization underlying bidirectional plasticity. Nat Neurosci 7:1104-1112.

Sans N, Petralia RS, Wang YX, Blahos J 2nd, Hell JW, Wenthold RJ (2000) A developmental change in NMDA receptor-associated proteins at hippocampal synapses. J Neurosci 20:1260-1271.

Sans N, Prybylowski K, Petralia RS, Chang K, Wang YX, Racca C, Vicini S, Wenthold RJ (2003) NMDA receptor trafficking through an interaction between PDZ proteins and the exocyst complex. Nat Cell Biol 5:520-530.

Schlüter OM, Xu W, Malenka RC (2006) Alternative N-terminal domains of PSD-95 and SAP97 govern activity-dependent regulation of synaptic AMPA receptor function. Neuron 51:99-111.

Tada T, Sheng M (2006) Molecular mechanisms of dendritic spine morphogenesis. Curr Opin Neurobiol 16:95-101.
Tarpey P, Parnau J, Blow M, Woffendin H, Bignell G, Cox C, Cox J, Davies H, Edkins S, Holden S, Korny A, Mallya U, Moon J, O’Meara S, Parker A, Stephens P, Stevens C, Teague J, Donnelly A, Mangelsdorf M, et al. (2004) Mutations in the DLG3 gene cause nonsyndromic X-linked mental retardation. Am J Hum Genet 75:318-324.

Tashiro A, Yuste R (2004) Regulation of dendritic spine motility and stability by Racl and Rho kinase: evidence for two forms of spine motility. Mol Cell Neurosci 26:429-440.

Tolias KF, Bikoff JB, Burette A, Paradis S, Harrar D, Tavazoie S, Weinberg RJ, Greenberg ME (2005) The Racl-GEF Tiaml couples the NMDA receptor to the activity-dependent development of dendritic arbors and spines. Neuron 45:525-538.

Topinka JR, Bredt DS (1998) N-terminal palmitoylation of PSD-95 regulates association with cell membranes and interaction with $\mathrm{K}^{+}$channel Kv1.4. Neuron 20:125-134.

van Zundert B, Yoshii A, Constantine-Paton M (2004) Receptor compartmentalization and trafficking at glutamate synapses: a developmental proposal. Trends Neurosci 27:428-437.

Washbourne P, Liu XB, Jones EG, McAllister AK (2004) Cycling of NMDA receptors during trafficking in neurons before synapse formation. J Neurosci 24:8253-8264.

Wenthold RJ, Prybylowski K, Standley S, Sans N, Petralia RS (2003) Trafficking of NMDA receptors. Annu Rev Pharmacol Toxicol 43:335-358.

Xie Z, Srivastava DP, Photowala H, Kai L, Cahill ME, Woolfrey KM, Shum CY, Surmeier DJ, Penzes P (2007) Kalirin-7 controls activity-dependent structural and functional plasticity of dendritic spines. Neuron 56:640-656.

Yoshihara Y, De Roo M, Muller D (2009) Dendritic spine formation and stabilization. Curr Opin Neurobiol 19:146-153.

Zanni G, van Esch H, Bensalem A, Saillour Y, Poirier K, Castelnau L, Ropers HH, de Brouwer AP, Laumonnier F, Fryns JP, Chelly J (2010) A novel mutation in the DLG3 gene encoding the synapse-associated protein 102 (SAP102) causes non-syndromic mental retardation. Neurogenetics 11:251-255.

Zhou Q, Homma KJ, Poo MM (2004) Shrinkage of dendritic spines associated with long-term depression of hippocampal synapses. Neuron 44: 749-757. 